\title{
Light Higgsinos as heralds of higher-dimensional unification
}

\section{F. Brümmer and W. Buchmüller}

Deutsches Elektronen-Synchrotron DESY, Notkestraße 85, D-22607 Hamburg, Germany

E-mail: felix.bruemmer@desy.de, buchmuwi@mail.desy.de

AbStract: Grand-unified models with extra dimensions at the GUT scale will typically contain exotic states with Standard Model charges and GUT-scale masses. They can act as messengers for gauge-mediated supersymmetry breaking. If the number of messengers is sizeable, soft terms for the visible sector fields will be predominantly generated by gauge mediation, while gravity mediation can induce a small $\mu$ parameter. We illustrate this hybrid mediation pattern with two examples, in which the superpartner spectrum contains light and near-degenerate higgsinos with masses below $200 \mathrm{GeV}$. The typical masses of all other superpartners are much larger, from at least $500 \mathrm{GeV}$ up to several $\mathrm{TeV}$. The lightest superparticle is the gravitino, which may be the dominant component of dark matter.

KeYwords: Supersymmetric Standard Model, Supersymmetry Breaking, Compactification and String Models

ARXIV EPRINT: 1105.0802 


\section{Contents}

1 Introduction 1

2 Soft terms from hybrid gauge-gravity mediation 3

3 Models $\quad 7$

3.1 A toy model $\quad 7$

3.2 A model from a heterotic orbifold 9

$\begin{array}{lll}3.3 & \text { Comments on multiple messenger scales } & 14\end{array}$

$\begin{array}{lll}3.4 & \text { Naturalness } & 15\end{array}$

4 Cosmology and phenomenology 16

5 Conclusions $\quad 18$

\section{Introduction}

Supersymmetry, extra space-time dimensions, and grand unification are among the most promising proposals for physics beyond the Standard Model. These three paradigms are elegantly incorporated in various modern approaches to unified model building, such as heterotic string compactifications, F-theory models, or purely field-theoretic orbifold GUTs. Extra dimensions opening up around the GUT scale provide new mechanisms to break the GUT gauge symmetry and to solve the problems of conventional four-dimensional SUSY GUTs, such as doublet-triplet splitting or proton decay. They are necessary ingredients in unifying the Standard Model forces with gravity within string theory.

The gauge couplings in the Minimal Supersymmetric Standard Model (MSSM) unify at $M_{\mathrm{GUT}} \approx 10^{16} \mathrm{GeV}$, which sets the scale where the GUT gauge symmetry should be broken and around which unwanted exotic states will decouple. In conventional fourdimensional GUTs, all states fall into complete GUT multiplets at scales above $M_{\mathrm{GUT}}$. This is in general not true in higher-dimensional constructions. In orbifold GUTs, for instance, the massless spectrum generally contains incomplete GUT multiplets. They can originate from the untwisted or twisted sector of an underlying heterotic string model. Likewise, F-theory GUTs can give rise to split multiplets when breaking the unified gauge group with hypercharge flux.

Obtaining a pair of Higgs doublets without their colour triplet partners is of course very welcome. Any additional split multiplets, however, should pick up masses not too far from $M_{\mathrm{GUT}}$ in realistic models, in order not to affect gauge coupling unification. ${ }^{1}$ This

\footnotetext{
${ }^{1}$ An exception here might be F-theory models, in which gauge couplings are not actually predicted to unify at $M_{\mathrm{GUT}}[1]$.
} 
can be achieved by Standard Model singlet fields acquiring expectation values of the order $M_{\mathrm{GUT}}$, thus giving masses to vector-like pairs of exotics. If these singlets also obtain $F$-term expectation values from coupling to the SUSY breaking hidden sector, the vectorlike exotics will act as messenger fields for gauge-mediated SUSY breaking. The resulting patterns of soft SUSY breaking terms will be rather different from that of conventional lowscale gauge mediation, where the messengers would need to form complete GUT multiplets. Here, with the messenger scale close to the GUT scale, the presence of split messenger multiplets generically leads to non-universal gaugino masses. ${ }^{2}$

Moreover, with a messenger scale $M_{\mathrm{GUT}}$, gauge-mediated contributions to soft SUSY breaking terms are comparable with gravity-mediated soft terms. This is because the latter are $M_{\mathrm{P}}$-suppressed, while the gauge-mediated terms are loop-suppressed, and the GUT scale is lower than the Planck scale by roughly a loop factor. For a sizeable number of messengers, gauge-mediated terms will dominate. Gauge mediation does not, however, give rise to a $\mu$ term (unless specific Higgs-messenger couplings are introduced, which in turn would induce a too large $B_{\mu}$ ). Instead $\mu$ can be generated, as in gravity mediation, by the Giudice-Masiero mechanism [4]. It will thus be of the order of the gravitino mass, and smaller than the soft masses if these are predominantly of gauge mediation origin. The same applies to $B_{\mu}$ and to the trilinear $a$-terms, which are suppressed in gauge mediation. The GUT-scale MSSM parameters are then characterised roughly by the hierarchy

$$
\left\{\mu, m_{3 / 2}, a, \sqrt{B_{\mu}}\right\} \ll\left\{M_{1 / 2}, m_{0}, m_{H}\right\} .
$$

Here $a$ stands collectively for any trilinear $a$-parameters, $m_{0}$ for squark and slepton soft masses, $m_{H}$ for Higgs soft masses, and $M_{1 / 2}$ for gaugino masses.

Current experimental limits from chargino searches are consistent with the "gravitymediated" terms on the 1.h.s. around $100 \mathrm{GeV}$, whereas the "gauge-mediated" terms on the r.h.s. may be $\mathrm{TeV}$ or larger. This leads to a quite peculiar low-energy spectrum with the following main features: The only light states with masses around $100 \mathrm{GeV}$ are Higgs and higgsino fields and the gravitino. The gravitino can naturally be the lightest supersymmetric particle (LSP), with a higgsino-like neutralino NLSP. The second neutralino and a higgsino-like chargino are slightly heavier. The mass of the lightest Higgs scalar is lifted to around $120 \mathrm{GeV}$ by large squark loop effects. All the remaining states, that is the heavier Higgs bosons, squarks, sleptons, gluinos, and gaugino-like chargino and neutralinos, have masses of at least around $500 \mathrm{GeV}$ and up to several $\mathrm{TeV}$ (the only possible exception being one of the scalar taus, which can be somewhat lighter).

The resulting LHC physics is quite rich and distinctive, and will be the subject of a future study. Here we merely point out that, in our scenario, most of the new MSSM particles are necessarily beyond the reach of early LHC searches. In particular, all coloured states are predicted to be rather heavy. This is consistent with the null result of the first LHC SUSY searches so far [5-10].

The stable gravitino serves as a natural dark matter candidate, allowing for a high reheating temperature as required by thermal leptogenesis. With standard cosmology and

\footnotetext{
${ }^{2}$ Similar observations were made in the context of F-theory models in [2]. More recently, in [3] messengers in split multiplets were used in heterotic models to improve the precision of gauge coupling unification.
} 
if R-parity is conserved, the higgsino NLSP is long-lived, and one has to make sure that its late-time decay does not destroy the successful predictions of primordial nucleosynthesis. Such a picture was first advocated in [11], and has recently been studied for a general neutralino LSP in [12]. In our case the NLSP abundance is significantly reduced by coannihilation with charginos, since the lighter chargino is nearly mass-degenerate with the NLSP. It turns out, however, that this effect alone is not sufficient to evade the stringent BBN bounds. As we shall see, our model can still be made consistent with early-universe cosmology with some mild modifications, such as a small amount of R-parity violation, or some moderate entropy production before nucleosynthesis.

\section{Soft terms from hybrid gauge-gravity mediation}

The simplest models of gauge-mediated supersymmetry breaking (see [13] for a review) contain a background chiral superfield $X$ which breaks supersymmetry, as well as some massive messenger fields in vector-like pairs $\Sigma_{i}, \widetilde{\Sigma}_{i}$. There is a superpotential

$$
W=\sum_{i} \lambda_{i} X \Sigma_{i} \widetilde{\Sigma}_{i}
$$

which, after $X$ is set to its expectation value $\langle X\rangle=M_{\mathrm{m}}+F \theta^{2}$, gives rise to both a supersymmetric messenger mass $M_{\mathrm{m}}$ and a SUSY-breaking mass splitting. Provided that $F \ll M_{\mathrm{m}}^{2}$, the contribution of each messenger pair to the gaugino masses at the messenger scale is

$$
M_{a}=\frac{g_{a}^{2}}{16 \pi^{2}} n_{a}\left(r_{i}\right) \frac{F}{M_{\mathrm{m}}} .
$$

Here $a=1,2,3$ labels the Standard Model gauge factors, $g_{a}$ is the running gauge coupling, and the messengers are in representations $r_{i}$ and $\bar{r}_{i}$ with Dynkin index $n_{a}\left(r_{i}\right)$.

In addition there is also a gravity-mediated contribution to the soft masses. For instance, if the gauge kinetic functions depend on $X$ as in

$$
\mathcal{L}=\frac{1}{4} \sum_{a} \int d^{2} \theta\left(\frac{1}{g_{a}^{2}}+\kappa_{a} \frac{X}{M_{\mathrm{P}}}\right) W^{a \alpha} W_{\alpha}^{a}+\text { h.c. },
$$

then there will be gravity-mediated terms

$$
M_{a}=\frac{1}{2} g_{a}^{2} \kappa_{a} \frac{F}{M_{\mathrm{P}}}
$$

with the Planck scale $M_{\mathrm{P}}=2.4 \cdot 10^{18} \mathrm{GeV} .^{3}$

For couplings $\kappa_{a}$ of order one, the contribution of each messenger pair is comparable with the gravity-mediated piece if the messenger masses are roughly a loop factor below the Planck scale. For a large number of messengers gauge mediation dominates.

\footnotetext{
${ }^{3}$ The $X$-dependence also affects the gauge coupling through the vev for the lowest component of $X$. We neglect this since we are interested in the case $M_{\mathrm{m}} / M_{\mathrm{P}} \ll 1$.
} 
Similar statements hold for soft scalar masses. The gauge-mediated soft mass for a chiral supermultiplet $\Phi$ is

$$
m_{\Phi}^{2}=\frac{2}{\left(16 \pi^{2}\right)^{2}}\left(\sum_{a i} g_{a}^{4} C_{a} n_{a}\left(r_{i}\right)\right)\left|\frac{F}{M_{\mathrm{m}}}\right|^{2},
$$

where $C_{a}$ is the quadratic Casimir for the representation under which $\Phi$ transforms. Gravity-mediated pieces are induced by operators

$$
\mathcal{L}=\int d^{4} \theta\left(\frac{X^{\dagger}}{M_{\mathrm{P}}}+\text { h.c. }-\frac{1}{2} \frac{X^{\dagger} X}{M_{\mathrm{P}}^{2}}\right) \Phi^{\dagger} \Phi,
$$

with $\mathcal{O}(1)$ couplings omitted, which gives

$$
m_{\Phi}^{2}=\frac{1}{2}\left|\frac{F}{M_{\mathrm{P}}}\right|^{2} .
$$

As for the gaugino masses, the typical mass scale for the contribution of each messenger pair is $F /\left(16 \pi^{2} M_{\mathrm{m}}\right)$, while the typical mass scale for the gravity-mediated piece is $F / M_{\mathrm{P}}$. They are comparable if $M_{\mathrm{m}} \approx M_{\mathrm{P}} /\left(16 \pi^{2}\right)$. Gauge mediation dominates if the number of messengers is sizeable. Note that scalar masses grow roughly with the square root of the messenger number; this is unlike the gaugino masses, which grow linearly with the messenger number and hence tend to be larger if the latter is large.

The remaining dimensionful MSSM parameters are the higgsino mass $\mu$, the Higgs mass mixing parameter $B_{\mu}$, and the trilinear $a$-parameters. We assume that a $M_{\mathrm{P} \text {-sized } \mu}$ at the renormalisable level is absent, and focus on how a suitable effective $\mu$ term is induced after supersymmetry breaking. It is well known that no $\mu$ and $B_{\mu}$ terms are generated in minimal gauge mediation. Gravity mediation, however, does generically generate them: For instance, terms of the form

$$
\mathcal{L}=\int d^{4} \theta \frac{X^{\dagger}}{M_{\mathrm{P}}} H_{u} H_{d}+\int d^{4} \theta \frac{X^{\dagger} X}{M_{\mathrm{P}}^{2}} H_{u} H_{d}+\text { h.c. }
$$

induce $\mu=\bar{F} / M_{\mathrm{P}}$ and $B_{\mu}=\left|F / M_{\mathrm{P}}\right|^{2}[4]$.

Trilinear $a$-terms in gauge mediation arise only at higher loop order, and are thus suppressed with respect to the gaugino masses. Provided that the number of messengers is not large enough to compensate for this loop suppression (which it never is in the models we are concerned with), the trilinear terms are predominantly generated by gravity mediation, $a \sim F / M_{\mathrm{P}}$.

The gravitino mass $m_{3 / 2}$, finally, is given by

$$
m_{3 / 2}=\frac{F}{\sqrt{3} M_{\mathrm{P}}}
$$

in Minkowski vacua. It is thus of the order of the gravity-mediated soft masses as usual.

If gauge-mediation is to be the dominant source for gaugino and scalar soft masses, there should be a sizeable number of messenger fields. Messenger multiplicities are bounded 
from above in conventional low-scale gauge mediation, since too many messengers would cause the gauge couplings to run into a Landau pole below the GUT scale. But in highscale models like ours this is clearly not a concern, as the messengers decouple already at the GUT-scale.

What can instead be a problem is the different scaling behaviour of scalar and gaugino masses with the messenger number. For illustration, consider a unified model containing $N_{5}$ pairs of $\mathbf{5} \oplus \overline{\mathbf{5}}$ messengers with GUT-scale masses. The gaugino masses grow as $N_{5}$, while the scalar masses grow as $\sqrt{N_{5}}$. For $N_{5}$ large enough that gauge mediation dominates, $N_{5} \gtrsim 5$ say, it becomes difficult to achieve realistic electroweak symmetry breaking. More precisely, if $M_{3} \gg m_{H_{u}}$ at the GUT scale, then $m_{H_{u}}^{2}$ will run down very quickly towards the low scale. This effect is caused mainly by loops of third generation squarks, whose masses in turn are driven large by gluino loop corrections. Thus $m_{H_{u}}^{2}$ at the electroweak scale will be large and negative, and should be compensated for by a similarly large $|\mu|^{2}$, as can best be seen from the relation

$$
-\frac{M_{Z}^{2}}{2} \simeq|\mu|^{2}+m_{H_{u}}^{2}
$$

valid for $\tan \beta \gtrsim 5$. Eq. (2.10) clearly requires a large cancellation on the r.h.s. in order to reproduce the measured value of $M_{Z} \approx 91 \mathrm{GeV}$. In our scenario $\mu$ is suppressed at the GUT scale, and will not change significantly through renormalisation group running. Therefore a large negative $m_{H_{u}}^{2}$ cannot be cancelled in eq. (2.10), so the gluino mass should not be large to begin with.

This problem can be solved in models where the messengers do not form complete GUT multiplets, and whose gaugino masses are therefore non-universal. Specifically, the messenger sector should comprise a large number of weak doublet messengers and comparatively few colour triplet pairs; this will lead to larger (positive) $m_{H_{u}}^{2}$ and smaller $M_{3}$ at the high scale. Again, in usual low-scale gauge mediation such a model would be incompatible with gauge coupling unification, but if the messengers decouple around the GUT scale, they will barely influence the evolution of the gauge couplings. One might object that, in the framework of conventional 4D GUTs, the essential feature of grand unification is precisely to have all states in complete GUT multiplets at and above the GUT scale. However, as pointed out in the Introduction, this is not the case in the framework of orbifold GUTs [14-20], or related heterotic orbifold models [21-26]. They, on the contrary, generically predict various states in split representations. Split multiplets are also common within a different class of string constructions, namely, F-theory GUTs [27-30]. In F-theory the GUT group group is broken via hypercharge flux, which leads to exotic states in incomplete GUT multiplets [31]. In fact it has been argued that such states might even be required in order to be consistent with the observed gauge coupling unification [1,31].

Our main example is motivated by the heterotic string construction of [25, 26]. It exhibits not only messengers in split representations, but even a pattern of precisely the sort that is favourable for electroweak symmetry breaking according to the above discussion: There are more weak doublets than colour triplets, hence the gluino mass is suppressed and the soft Higgs masses are enhanced. 
One of the main reasons why low-scale gauge mediation is considered to be attractive is that it generates flavour-blind soft terms. This is still the case in our scenario, but as opposed to the low-scale case, here the gravity-mediated contributions to soft terms are sizeable. They will generically induce unacceptably large flavour-changing neutral currents. ${ }^{4}$ To suppress flavour violation in the gravity-mediated soft terms, an additional mechanism is necessary - for instance, wave-function localisation or horizontal symmetries.

A fairly robust prediction of our scenario is large $\tan \beta$ : The $B_{\mu}$ parameter is induced by gravity mediation and therefore suppressed with respect to $m_{H_{d}}^{2}$. Thus

$$
\tan \beta \simeq \frac{m_{H_{u}}^{2}+m_{H_{d}}^{2}+2|\mu|^{2}}{2 B_{\mu}}
$$

is large at the high scale. This remains true after running to the electroweak scale, since $B_{\mu}$ cannot grow large if $\mu$ is small, and since $m_{H_{d}}^{2}$ cannot shrink significantly unless $\tan \beta$ is large.

A related feature is a scalar $\tau$ lepton which is relatively light compared to the remaining squarks and sleptons. In fact we will see that, when we discuss concrete models, squark and slepton masses will be around at least half a $\mathrm{TeV}$, while the higgsino-like neutralinos and chargino will have masses around $100-200 \mathrm{GeV}$. The lighter $\tilde{\tau}$ can have a mass somewhere in between: Since $\tilde{\tau}_{R}$ only has hypercharge gauge interactions, the RG evolution of $m_{\tilde{\tau}_{R}}$ will be mainly driven by the $\tau$ Yukawa coupling to the Higgs sector, which is large at large $\tan \beta$ and drives $m_{\tilde{\tau}_{R}}$ down.

Other characteristics of the low-energy spectrum include near-degenerate masses for the light neutralinos and chargino (with mass differences of the order of a few $\mathrm{GeV}$ ), and a similar mass degeneracy for the heaviest neutralino and the heavier charginos. This is because the former are almost completely higgsinos, while the latter are almost completely Winos. Furthermore, the heavier neutral Higgs scalar is almost degenerate with the pseudoscalar Higgs and close in mass to the charged Higgs, since we are in an extreme decoupling limit.

It is worthwile to briefly compare our approach with the scenario dubbed "sweet spot supersymmetry" in [36], which has also been argued to be realised in F-theory [37], and where the MSSM soft parameters and $\mu$ are also generated from a combination of high-scale gauge mediation and higher-dimensional operators. The authors of [36] propose gaugemediated SUSY breaking with $m_{3 / 2} \approx 1 \mathrm{GeV}$, roughly corresponding to $F \approx\left(10^{9} \mathrm{GeV}\right)^{2}$ and $M_{\mathrm{m}} \approx 10^{13} \mathrm{GeV}$. Furthermore they assume that $\mu$ and $B_{\mu}$ are generated by higherdimensional operators whose suppression scale $\Lambda$ is below the Planck scale. For instance, the Higgs fields could couple directly to the hidden sector, which would induce the operators

$$
\mathcal{L}=\int d^{4} \theta \frac{X^{\dagger}}{\Lambda} H_{u} H_{d}+\text { h.c. }+\int d^{4} \theta \frac{X^{\dagger} X}{\Lambda^{2}}\left(H_{u}^{\dagger} H_{u}+H_{d}^{\dagger} H_{d}\right)
$$

after integrating out certain hidden sector states of mass $\Lambda$. For $\Lambda \approx 10^{16} \mathrm{GeV}$ eq. (2.12) gives an electroweak-scale $\mu$ term, as well as sizeable contributions to the Higgs soft masses.

\footnotetext{
${ }^{4}$ See e.g. [32-35] for recent studies of the flavour problem in hybrid gauge-gravity mediation.
} 
Such models may thus be viewed as standard gauge mediation models with two unusual features: The $\mu$ problem is solved by directly coupling the Higgs fields to the hidden sector, and the gravitino is a viable dark matter candidate. By construction, gravity mediation proper (i.e. visible sector soft terms induced by $M_{\mathrm{P}}$-suppressed operators) never plays a role. Indeed similar models could be constructed with lower $m_{3 / 2}$ and correspondingly lower $\Lambda, F$, and $M_{\mathrm{m}}$, if one gives up the requirement that the gravitino should be dark matter.

Our scenario, by contrast, requires the introduction of just a single new scale: the scale of supersymmetry breaking $\sqrt{F} \approx 10^{10} \mathrm{GeV}$. The messenger mass is given by the GUT scale or compactification scale, and the suppression scale for the $\mu$ term is fixed to be $M_{\mathrm{P}}$. We consider this quite appealing from the model-building point of view. However, in our restricted framework there is naturally less freedom to evade experimental constraints by simply adjusting the scales. For instance, as previously stated, in our scenario the flavour problem is not automatically solved, but requires some extra mechanism.

Note also that our models are very different from hybrid gauge-gravity mediation with anomalous U(1)s where all messenger fields are in complete GUT multiplets [38, 39]. While such models also allow for gravitino dark matter, this requires all other superparticle masses to be at least around a $\mathrm{TeV}[40]$.

Concerning the superparticle spectrum, our models share some characteristic features with "lopsided gauge mediation" [41], which also predicts light higgsinos along with coloured states heavier than a TeV. However, in contrast to our models, the sleptons and the bino still tend to be relatively light in the models of [41], while the pseudoscalar Higgs is extremely heavy. The theoretical framework is, of course, also very different, lopsided gauge mediation essentially being pure gauge mediation with certain direct Higgs-messenger couplings.

\section{Models}

\subsection{A toy model}

Let us first present a model whose purpose it is to illustrate our scenario in the simplest possible way, without being motivated by any specific UV completion. Consider $N_{5}$ pairs of messengers in the $\mathbf{5} \oplus \overline{\mathbf{5}}$ of $\mathrm{SU}(5)$, and $N_{2}$ pairs of messengers in the $\mathbf{2}_{1 / 2} \oplus \mathbf{2}_{-1 / 2}$ of $\mathrm{SU}(2)_{L} \times \mathrm{U}(1)_{Y}$. We take all messenger masses to be equal, $M_{\mathrm{m}}=10^{16} \mathrm{GeV}$, and $F=\left(2 \cdot 10^{10} \mathrm{GeV}\right)^{2}$, corresponding to $m_{3 / 2}=100 \mathrm{GeV}$. Furthermore we set

$$
\begin{aligned}
\mu & \sim \frac{F}{M_{\mathrm{P}}}, \\
A_{0} & \sim \frac{F}{M_{\mathrm{P}}}, \\
B_{\mu} & \sim \frac{F^{2}}{M_{\mathrm{P}}^{2}}
\end{aligned}
$$

at $M_{\mathrm{GUT}}$, to account for these terms being generated by gravity mediation. Here $A_{0}$ is, as usual, related to the trilinear $a$-parameters by

$$
a_{u, d, l}=A_{0} y_{u, d, l}
$$

with $y_{u, d, l}$ the Yukawa matrices. 
The gauge-mediated contributions to the gaugino masses are

$$
\begin{aligned}
& M_{3}=\frac{g^{2}}{16 \pi^{2}} N_{5} \frac{F}{M_{\mathrm{m}}}, \\
& M_{2}=\frac{g^{2}}{16 \pi^{2}}\left(N_{5}+N_{2}\right) \frac{F}{M_{\mathrm{m}}}, \\
& M_{1}=\frac{g^{2}}{16 \pi^{2}}\left(N_{5}+\frac{3}{5} N_{2}\right) \frac{F}{M_{\mathrm{m}}} .
\end{aligned}
$$

For the scalar masses, we obtain

$$
\begin{aligned}
m_{Q}^{2} & =\left(\frac{g^{2}}{16 \pi^{2}}\right)^{2}\left(\frac{F}{M_{\mathrm{m}}}\right)^{2}\left(\frac{21}{5} N_{5}+\frac{38}{25} N_{2}\right), \\
m_{U}^{2} & =\left(\frac{g^{2}}{16 \pi^{2}}\right)^{2}\left(\frac{F}{M_{\mathrm{m}}}\right)^{2}\left(\frac{16}{5} N_{5}+\frac{8}{25} N_{2}\right), \\
m_{D}^{2} & =\left(\frac{g^{2}}{16 \pi^{2}}\right)^{2}\left(\frac{F}{M_{\mathrm{m}}}\right)^{2}\left(\frac{14}{5} N_{5}+\frac{2}{25} N_{2}\right), \\
m_{L}^{2}=m_{H_{u}}^{2}=m_{H_{d}}^{2} & =\left(\frac{g^{2}}{16 \pi^{2}}\right)^{2}\left(\frac{F}{M_{\mathrm{m}}}\right)^{2}\left(\frac{9}{5} N_{5}+\frac{42}{25} N_{2}\right), \\
m_{E}^{2} & =\left(\frac{g^{2}}{16 \pi^{2}}\right)^{2}\left(\frac{F}{M_{\mathrm{m}}}\right)^{2}\left(\frac{6}{5} N_{5}+\frac{18}{25} N_{2}\right) .
\end{aligned}
$$

Neglecting gravity-mediated contributions to scalar soft masses and gaugino masses altogether, it is possible to obtain realistic electroweak symmetry breaking e.g. with the following parameters:

$$
\begin{aligned}
N_{5} & =6 \\
N_{2} & =10 \\
\mu & =145 \mathrm{GeV}, \\
A_{0} & =145 \mathrm{GeV}, \\
B_{\mu} & =(223 \mathrm{GeV})^{2} .
\end{aligned}
$$

The gauge-mediated soft masses at $M_{\mathrm{GUT}}=1.2 \cdot 10^{16} \mathrm{GeV}$ are then as in table 1 . The low-energy particle spectrum as computed with SOFTSUSY [42] is shown in table 2. As expected $\tan \beta$ turns out to be rather large, $\tan \beta=48$. Generally the spectrum is much as anticipated in section 2. There is a Standard Model-like Higgs with its mass just above the LEP bound. The higgsino-like neutralinos and chargino are light and almost degenerate in mass. Except for a relatively light $\tilde{\tau}$, all other states have masses above $600 \mathrm{GeV}$ and up to more than $2 \mathrm{TeV}$. The heavier Higgs (pseudo)scalars have very similar masses, and also the heavier chargino is nearly degenerate with the heaviest neutralino. 


\begin{tabular}{|c|c|}
\hline mass parameter & value $[\mathrm{GeV}]$ \\
\hline \hline$M_{1}$ & 1592 \\
$M_{2}$ & 2122 \\
$M_{3}$ & 796 \\
\hline$m_{Q}$ & 843 \\
$m_{U}$ & 627 \\
$m_{D}$ & 556 \\
\hline$m_{L}=m_{H_{u}}=m_{H_{d}}$ & 697 \\
$m_{E}$ & 503 \\
\hline
\end{tabular}

Table 1. GUT-scale parameters for a toy model with $N_{5}=6$ pairs of 5-plet messengers and $N_{2}=10$ pairs of doublet messengers.

\begin{tabular}{|c|c|}
\hline particle & mass $[\mathrm{GeV}]$ \\
\hline \hline$h_{0}$ & 118 \\
\hline$\chi_{1}^{0}$ & 124 \\
$\chi_{1}^{ \pm}$ & 127 \\
$\chi_{2}^{0}$ & 130 \\
\hline$\chi_{3}^{0}$ & 691 \\
$\chi_{4}^{0}$ & 1724 \\
$\chi_{2}^{ \pm}$ & 1724 \\
\hline$H_{0}$ & 764 \\
$A_{0}$ & 765 \\
$H^{ \pm}$ & 770 \\
\hline$\tilde{g}$ & 1792 \\
\hline$\tilde{\tau}_{1}$ & 211 \\
other sleptons & $780-1550$ \\
\hline squarks & $1090-2180$ \\
\hline
\end{tabular}

Table 2. Low-energy spectrum for the mass parameters of table 1 , with $\mu=A_{0}=145 \mathrm{GeV}$ and $B_{\mu}=(223 \mathrm{GeV})^{2}$.

\subsection{A model from a heterotic orbifold}

Higher-dimensional orbifold GUTs, or heterotic string compactifications, naturally contain incomplete GUT multiplets. These can arise from twisted states, localised at orbifold singularities where only a subgroup of the GUT group is realised; or from untwisted states whose zero modes are partly projected out by the orbifold. In addition, there are generally also massless pairs of vector-like exotics in complete GUT representations. All of these exotics should eventually become massive, leaving only a pair of light Higgs doublets and the three generations of chiral MSSM matter at low energies. In principle a limited number of complete GUT multiplets could survive down to energies far below $M_{\mathrm{GUT}}$, without affecting 


\begin{tabular}{|c|c|c|c|}
\hline field & representation & multiplicity & 6D origin \\
\hline$d$ & $(\mathbf{3}, \mathbf{1})_{-1 / 3}$ & 4 & bulk \\
$\tilde{d}$ & $(\overline{\mathbf{3}}, \mathbf{1})_{1 / 3}$ & 4 & bulk \\
$\ell$ & $(\mathbf{1}, \mathbf{2})_{1 / 2}$ & 4 & bulk \\
$\tilde{\ell}$ & $(\mathbf{1}, \mathbf{2})_{-1 / 2}$ & 4 & bulk \\
$m$ & $(\mathbf{1}, \mathbf{2})_{0}$ & 8 & brane \\
$s^{+}$ & $(\mathbf{1}, \mathbf{1})_{1 / 2}$ & 16 & brane \\
$s^{-}$ & $(\mathbf{1}, \mathbf{1})_{-1 / 2}$ & 16 & brane \\
\hline
\end{tabular}

Table 3. The messenger content of a heterotic orbifold model.

perturbative gauge coupling unification. Here, however, we assume for simplicity a common mass for all messengers, which should then be close to $M_{\mathrm{GUT}}$ (see also section 3.3).

There are two ways in which the GUT scale can enter in heterotic orbifolds. First, usually some Standard Model singlets need to take vacuum expectation values in order to cancel the FI term of an anomalous U(1), whose magnitude is about a loop factor below $M_{\mathrm{P}}$. This introduces the scale $M_{\mathrm{P}} /\left(16 \pi^{2}\right) \approx M_{\mathrm{GUT}}$ into the scalar potential (similar as in the related string-inspired models of $[38,39])$. Second, MSSM gauge coupling unification motivates considering anisotropic compactifications [43, 44], where one or two of the radii of the internal manifold are large in string units (around $1 / M_{\mathrm{GUT}}$ ) while the others are small (around $1 / M_{\mathrm{P}}$ ). One obtains an intermediate effective description between $M_{\mathrm{GUT}}$ and $M_{\mathrm{P}}$ in terms of a $5 \mathrm{D}$ or $6 \mathrm{D}$ orbifold GUT. In this picture, the compactification of the larger two extra dimensions breaks the $5 \mathrm{D}$ or $6 \mathrm{D}$ bulk gauge symmetry to the Standard Model. Such a large compactification radius may again have its dynamical origin in the scale of the FI term, depending on the moduli stabilisation mechanism [45].

Our prime example for this section will be the model of [26]. The massless spectrum contains various chiral supermultiplets, both Standard Model singlets and fields with Standard Model charges. Besides the three generations of quarks and leptons, and a massless pair of Higgs doublets, there are several vector-like exotics which become massive when some of the singlet fields acquire vevs. The massless vector-like exotics are listed in table 3 , where we have also indicated the geometric origin of the zero modes in a $6 \mathrm{D}$ orbifold GUT limit. Note that in the present work we are restricting ourselves to the zero modes of the compactification, despite the fact that there is not a large separation between their masses and the Kaluza-Klein scale. In general, higher KK modes originating from bulk states will act as additional messengers, and their contributions should be taken into account in a more detailed study.

The messenger content of table 3 does not lead to realistic electroweak symmetry breaking when coupled to a hidden sector in the minimal manner as in the model of section 3.1, with a single goldstino background field and a common mass scale for all messengers. However, such a minimal setup is inappropriate for the present model for two reasons. First, the effective messenger-goldstino couplings (the $\lambda_{i}$ in eq. (2.1)) are expected to be different for different types of messengers. We will comment on possible consequences 
of this in section 3.3. Second, different messengers with their different geometric origins have distinct transformation properties under discrete symmetries. In our case the selection rules turn out to be such that there cannot be a single goldstino multiplet coupling to both bulk and brane fields. The next simplest option, therefore, is to introduce two SUSYbreaking background superfields $X_{1}$ and $X_{2}$ with couplings

$$
W=X_{1} d \tilde{d}+X_{1} \ell \tilde{\ell}+X_{2} m m+X_{2} s^{+} s^{-} .
$$

For simplicity we take their expectation values to be equal in the lowest component, but we do allow their $F$-terms to be distinct:

$$
\left\langle X_{1}\right\rangle=M_{\mathrm{m}}+F_{1} \theta^{2}, \quad\left\langle X_{2}\right\rangle=M_{\mathrm{m}}+F_{2} \theta^{2} .
$$

We can define a goldstino mixing angle $\phi$ by

$$
\begin{gathered}
F_{1}=F \cos \phi, \quad F_{2}=F \sin \phi, \\
\frac{F}{\sqrt{3} M_{\mathrm{P}}}=m_{3 / 2} .
\end{gathered}
$$

This gives for the gauge-mediated gaugino masses at the scale $M_{\mathrm{m}}$

$$
\begin{aligned}
M_{1} & =\frac{g^{2}}{16 \pi^{2}} \frac{F}{M_{\mathrm{m}}}\left(4 \cos \phi+\frac{24}{5} \sin \phi\right), \\
M_{2} & =\frac{g^{2}}{16 \pi^{2}} \frac{F}{M_{\mathrm{m}}}(4 \cos \phi+4 \sin \phi), \\
M_{3} & =\frac{g^{2}}{16 \pi^{2}} \frac{F}{M_{\mathrm{m}}} 4 \cos \phi,
\end{aligned}
$$

and for the scalar soft masses-squared

$$
\begin{aligned}
& m_{Q}^{2}=2\left(\frac{g^{2}}{16 \pi^{2}}\right)^{2}\left(\frac{F}{M_{\mathrm{m}}}\right)^{2}\left(\frac{287}{50}+\frac{133}{50} \cos 2 \phi\right), \\
& m_{U}^{2}=2\left(\frac{g^{2}}{16 \pi^{2}}\right)^{2}\left(\frac{F}{M_{\mathrm{m}}}\right)^{2}\left(\frac{96}{25}+\frac{64}{25} \cos 2 \phi\right), \\
& m_{D}^{2}=2\left(\frac{g^{2}}{16 \pi^{2}}\right)^{2}\left(\frac{F}{M_{\mathrm{m}}}\right)^{2}\left(\frac{74}{25}+\frac{66}{25} \cos 2 \phi\right), \\
& m_{L}^{2}=m_{H_{u}}^{2}=m_{H_{d}}^{2}=2\left(\frac{g^{2}}{16 \pi^{2}}\right)^{2}\left(\frac{F}{M_{\mathrm{m}}}\right)^{2}\left(\frac{183}{50}-\frac{3}{50} \cos 2 \phi\right), \\
& m_{E}^{2}=2\left(\frac{g^{2}}{16 \pi^{2}}\right)^{2}\left(\frac{F}{M_{\mathrm{m}}}\right)^{2}\left(\frac{66}{25}-\frac{6}{25} \cos 2 \phi\right) .
\end{aligned}
$$

In a $6 \mathrm{D}$ orbifold GUT picture, the messenger mass $M_{\mathrm{m}}$ should take a value around the scale of the two larger radii, which is slightly below the $4 \mathrm{D}$ unification scale. For concreteness we set $M_{\mathrm{m}}=5 \cdot 10^{15} \mathrm{GeV}$. Then $g$ is still approximately given by the unified gauge coupling at the GUT scale, $g \approx 0.7$. We again choose $F=\left(2 \cdot 10^{10} \mathrm{GeV}\right)^{2}$, so the gravitino mass is $m_{3 / 2}=100 \mathrm{GeV}$. 


\begin{tabular}{|c|c|}
\hline mass parameter & value $[\mathrm{GeV}]$ \\
\hline \hline$M_{1}$ & 1771 \\
$M_{2}$ & 1583 \\
$M_{3}$ & 644 \\
\hline$m_{Q}$ & 786 \\
$m_{U}$ & 599 \\
$m_{D}$ & 478 \\
\hline$m_{L}=m_{H_{u}}=m_{H_{d}}$ & 736 \\
$m_{E}$ & 643 \\
\hline
\end{tabular}

Table 4. GUT-scale parameters for the heterotic model of section 3.2, with $\cos \phi=0.466$ and a universal gravity-mediated piece $m_{0}=150 \mathrm{GeV}$.

To investigate this model, we now take into account both gauge-mediated and gravitymediated contributions to soft terms. To account for gravity mediation, we add a universal piece $m_{0}$ to the gaugino masses of eqs. (3.9), and to scalar masses in quadrature. Choosing

$$
m_{0}=150 \mathrm{GeV}=0.9 \frac{F}{M_{\mathrm{P}}}
$$

and setting

$$
\begin{aligned}
\tan \phi & =1.9, \\
\mu & =m_{0}, \\
A_{0} & =m_{0}, \\
B_{\mu} & =\left(1.6 m_{0}\right)^{2}=(240 \mathrm{GeV})^{2},
\end{aligned}
$$

the high-scale mass parameters are as listed in table 4 . These parameter values lead to realistic electroweak symmetry breaking at $\tan \beta=41$ and a low-energy spectrum as in table 5. The renormalisation group evolution of some selected parameters between the messenger scale and the $\mathrm{TeV}$ scale is depicted in figures 1 and 2 . In these plots, $M_{\mathrm{SUSY}}=1.17 \mathrm{TeV}$ is the scale where the Higgs potential is minimised, chosen to be the geometric mean of the stop masses. Figure 2 clearly shows the discrepancy between the gravity-mediated $\mu$ and $B_{\mu}$ on the one hand, and the gauge-mediated $m_{H_{d}}^{2}$ and $m_{H_{u}}^{2}$ on the other hand. Through its renormalisation group evolution, $\left|m_{H_{u}}^{2}\right|$ eventually becomes comparable with $|\mu|^{2}$ at $M_{\mathrm{SUSY}}$, to produce an electroweak symmetry breaking scale which is of the order of the gravity-mediated terms.

It is interesting that in this model the lighter $\tilde{\tau}$ is still relatively heavy, especially when compared to the model of section 3.1. This is due to the smaller $\tan \beta$ in the current setup, as well as to the presence of a large number of hypercharged messengers which increase the GUT-scale value of $m_{E}$ relative to the other masses. Otherwise the spectrum is qualitatively very similar to that in section 3.1.

Regarding the fundamental input parameters, note that the choice $\phi>\frac{\pi}{4}$ (i.e. $\tan \phi>$ 1) serves to further suppress $M_{3}$ with respect to $M_{2}$ and $M_{1}$. As discussed in section 2, a too 


\begin{tabular}{|c|c|}
\hline particle & mass $[\mathrm{GeV}]$ \\
\hline \hline$h_{0}$ & 117 \\
\hline$\chi_{1}^{0}$ & 137 \\
$\chi_{1}^{ \pm}$ & 140 \\
$\chi_{2}^{0}$ & 144 \\
\hline$\chi_{3}^{0}$ & 799 \\
$\chi_{4}^{0}$ & 1296 \\
$\chi_{2}^{ \pm}$ & 1296 \\
\hline$H_{0}$ & 856 \\
$A_{0}$ & 857 \\
$H^{ \pm}$ & 861 \\
\hline$\tilde{g}$ & 1453 \\
\hline$\tilde{\tau}_{1}$ & 713 \\
other sleptons & $910-1290$ \\
\hline squarks & $950-1750$ \\
\hline
\end{tabular}

Table 5. Low-energy spectrum for the mass parameters of table 4 , with $\mu=A_{0}=150 \mathrm{GeV}$ and $B_{\mu}=(240 \mathrm{GeV})^{2}$.

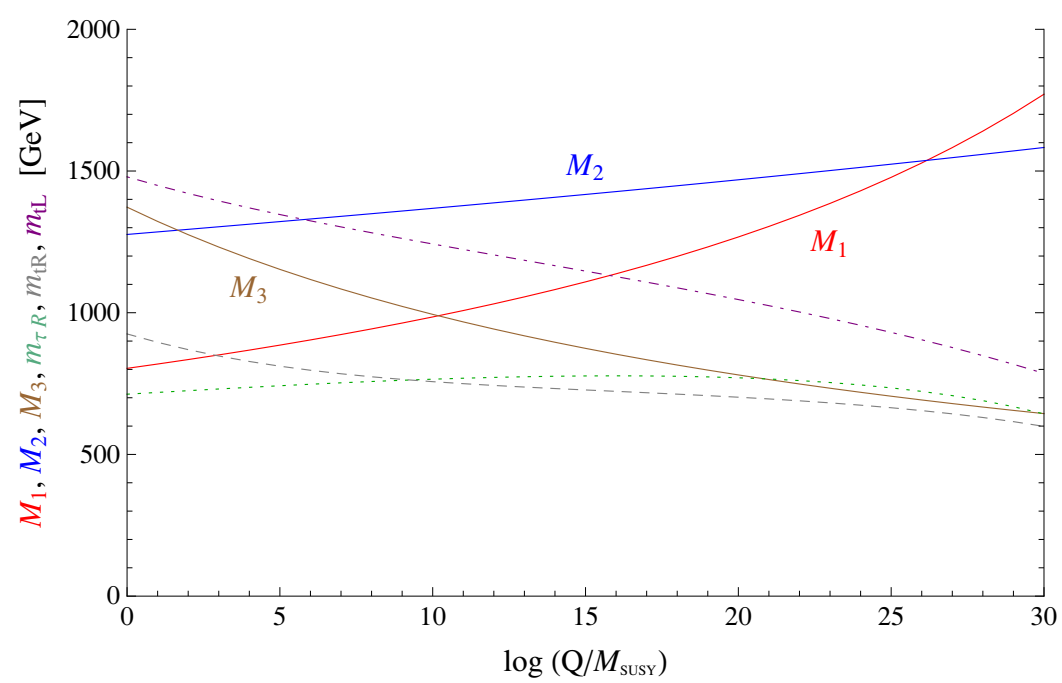

Figure 1. Two-loop renormalisation group evolution of gaugino masses and some selected sfermion masses for the model of section 3.2. The horizontal axis ranges between $M_{\mathrm{SUSY}}=1.17 \mathrm{TeV}$ on the left and the messenger scale $M_{\mathrm{m}}=5 \cdot 10^{15} \mathrm{GeV}$ on the right. Bino, wino and gluino masses are shown in red, blue, and brown. The green dotted curve shows the right-handed stau soft mass; the grey dashed and purple dot-dashed curves are the right-handed and left-handed stop soft masses.

large gluino mass would be in conflict with electroweak symmetry breaking. This problem is now solved in our model in a twofold way: First, there are more messengers charged under $\mathrm{SU}(2)_{L} \times \mathrm{U}(1)_{Y}$ than under $\mathrm{SU}(3)_{C}$; and second, the former couple somewhat more strongly to the hidden sector than the latter. 


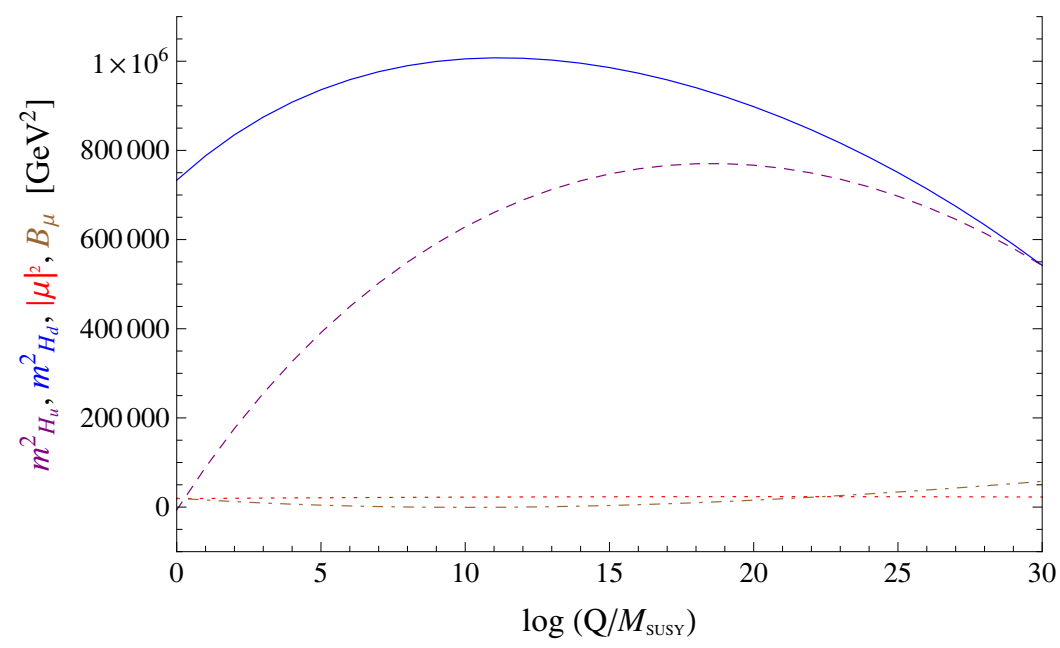

Figure 2. Renormalisation group evolution of Higgs mass parameters for the model of section 3.2. The horizontal axis is as in figure 1. The blue solid curve and the purple dashed curve show $m_{H_{d}}^{2}$ and $m_{H_{u}}^{2}$. The red dotted curve shows $|\mu|^{2}$, and the brown dot-dashed curve shows $B_{\mu}$.

\subsection{Comments on multiple messenger scales}

In the previous section we have assumed a common messenger mass scale $M_{\mathrm{m}}$ for all messenger fields. It would be more realistic to take into account that different messenger fields will, in fact, decouple at different scales. More precisely, in the heterotic orbifold model the messenger superpotential is, to quadratic order in the messengers $\Sigma_{i}, \widetilde{\Sigma}_{i}$,

$$
W=\sum_{i} M_{\mathrm{P}} \mathcal{P}_{i}\left(\frac{S_{I}}{M_{\mathrm{P}}}\right) \Sigma_{i} \widetilde{\Sigma}_{i}
$$

Here the $\mathcal{P}_{i}$ are certain polynomials of degree $>1$, and the $S_{I}$ are Standard Model singlet fields. The dimensionless coefficients entering the $\mathcal{P}_{i}$ are unknown (but could in principle be computed from the worldsheet CFT amplitudes of the underlying string model). The expectation values of the $S_{I}$ are also unknown, but a naive estimate gives $\left\langle S_{I}\right\rangle \approx M_{\mathrm{GUT}}$, which would be the correct order of magnitude to cancel a $M_{\mathrm{GUT}}$-sized FI term. Some of the $S_{I}$ should also acquire $F$-term vevs from coupling to the hidden sector. Denoting by $n_{i}$ the degree of the lowest non-vanishing monomial in $\mathcal{P}_{i}$, and assuming that all unknown coefficients are generic and of order unity, one obtains an effective superpotential resembling eq. (2.1):

$$
W=\sum_{i} \lambda_{i} X_{i} \Sigma_{i} \widetilde{\Sigma}_{i}
$$

where $\lambda_{i} \sim\left(\frac{M_{\mathrm{GUT}}}{M_{\mathrm{P}}}\right)^{n_{i}-1}$, and $\left\langle X_{i}\right\rangle \sim M_{\mathrm{GUT}}+F \theta^{2}$.

It is evident that, when taking these naive arguments seriously, messengers which couple to higher powers of the $S_{I}$ decouple at lower scales, while their gauge-mediated contribution to soft masses remains unchanged. Strictly speaking, however, our reasoning can at most serve as a rough indication that the messenger scales in this model can be 
hierarchically different. To treat this matter quantitatively, one would have to undertake the formidable task of computing the unknown couplings between the light modes, and subsequently minimising the full scalar potential.

Let us therefore merely point out how multiple messenger scales could help to render the model viable phenomenologically. Messengers in incomplete GUT multiplets, such as the $m$ and $s^{ \pm}$fields, should still decouple around the GUT scale in order not to interfere with gauge coupling unification. On the other hand, the $d, \tilde{d}, \ell$ and $\tilde{\ell}$ fields happen to fall into complete $\mathbf{5} \oplus \overline{\mathbf{5}}$ pairs (even though they do not originate from the same GUT multiplets in the string model), so their decoupling scale can be much lower. ${ }^{5}$ This is the case, in particular, if the superpotential has the structure of eq. (3.14), with the $\lambda_{i}$ very small for the $d$ - and $\ell$-type messengers. (Note again that the messenger masses depend on the $\lambda_{i}$, while the soft terms at the respective messenger scales do not.) Low messenger scales for the coloured messengers can now alleviate the problem which we pointed out in section 2 , namely, that large gluino masses tend to spoil electroweak symmetry breaking via their effect on renormalisation group running. In our model gauge-mediated gluino masses are induced only by $d$ and $\tilde{d}$ messengers. Therefore, they will be generated at low scales (if the $d$ and $\tilde{d}$ decouple at low energies), and hence will have less influence on the running of the Higgs mass parameters.

\subsection{Naturalness}

From figure 2 it is evident that some amount of fine-tuning is required in our model in order to reproduce realistic electroweak symmetry breaking. Namely, the input parameters have to be chosen such that $\left|m_{H_{u}}^{2}\right|$ is very small at $M_{\mathrm{SUSY}}$ for eq. (2.10) to be satisfied.

A quantitative measure of fine-tuning [46] is the maximum sensitivity of $M_{Z}$ with respect to variations of any of the continuous parameters,

$$
c=\max _{a \in\{\text { Parameters }\}}\left|\frac{\partial \log M_{Z}}{\partial \log a}\right| .
$$

Taking the set of parameters to be the MSSM parameters of table 4, along with $\mu$ and $B_{\mu}$, we find for the models of section 3.1 and section $3.2 c$-values of around $c \sim 200$, so the models could be considered to be fine-tuned at a level comparable with the CMSSM in its remaining viable parameter regions.

However, one should bear in mind that for our class of models, the dimensionful MSSM parameters are derived quantities and cannot be varied independently. A more appropriate set of fundamental parameters for the model of section 3.1 would thus be $\left\{F / M_{\mathrm{m}}, A_{0}, \mu, \sqrt{B_{\mu}}\right\}$, in addition to the discrete messenger numbers which do not enter in the definition of $c$, eq. (3.15). Computing the $c$-value with respect to these parameters, one obtains $c \sim 20$, which is a significant improvement (albeit still not quite $\mathcal{O}(1)$ ). This can be understood as follows: Gauge-mediated contributions to soft terms are large, and are governed by discrete parameters, while gravity-mediated contributions are small, and are determined by continuous parameters. Furthermore, if $\mu$ is small as in our case, then

\footnotetext{
${ }^{5}$ Indeed the $n_{i}$ are found to be large precisely for these fields in [26], but as already stated it would be premature to infer that they are correspondingly lighter.
} 
$M_{2}$ and $M_{3}$ are the MSSM soft terms to which the electroweak scale is by far the most sensitive. The fine-tuning is considerably reduced once we fix the ratio of the gauge-mediated contributions to $M_{2}$ and $M_{3}$ by some suitable choice of messenger numbers. Variations of the gravity-mediated terms do not have a large impact as long as gravity mediation is subdominant, and varying the overall scale of gauge mediation leads to a simultaneous change in both $M_{2}$ and $M_{3}$, the effects of which can (at least partially) cancel. The overall picture is similar to the one advocated in [47], where it was recently found that, quite generally, certain choices of non-universal gaugino mass ratios can help to reduce fine-tuning.

For the model of section 3.2 the fundamental continuous parameters are $\left\{F_{1} / M_{\mathrm{m}}\right.$, $\left.F_{2} / M_{\mathrm{m}}, m_{0}, \sqrt{B_{\mu}}\right\}$. Since here the gauge-mediated contributions to $M_{2}$ and $M_{3}$ still depend on a continuous parameter (the ratio $F_{1} / F_{2}$, or equivalently the goldstino angle $\phi$ ), the $c$-value with respect to this parameter set remains high.

Beyond these simple considerations, the naturalness question should probably be best left to the framework of a concrete UV completion which more rigorously defines the set of independent parameters. Of course, ultimately all parameters in the String Landscape will be discrete, so in that context even the entire notion of fine-tuning and naturalness here appears questionable.

\section{Cosmology and phenomenology}

A gravitino LSP with a mass of $\mathcal{O}(100) \mathrm{GeV}$ is an interesting dark matter candidate. At high reheating temperatures, as required by thermal leptogenesis, thermal production of gravitinos yields the observed dark matter abundance for typical gluino masses. A potential problem, however, are the severe constraints from BBN. The NLSP is long-lived, and its late decays inject highly energetic particles into the plasma after nucleosynthesis. These will destroy the newly formed nuclei and thus distort the successfully predicted light element abundances.

In the analysis of [11] this problem could be avoided for a sufficiently short lived higgsino NLSP since at that time the BBN constraints only imposed the upper bound on the higgsino abundance $\Omega_{\tilde{h}} h^{2} \lesssim 8 \cdot 10^{-3}$ for lifetimes $\tau_{\tilde{h}} \lesssim 2 \cdot 10^{6} \mathrm{~s}$ [48]. For a higgsino NLSP in the mass range $80 \mathrm{GeV}<m_{\tilde{h}}<300 \mathrm{GeV}$ the above $\mathrm{BBN}$ bound on the higgsino abundance is satisfied due to the effect of coannihilations $[49,50]$ and the lifetime constraint can be satisfied for gravitino masses below $100 \mathrm{GeV}$. Hence, a consistent picture of leptogenesis, gravitino dark matter and nucleosynthesis could be obtained.

The present BBN bounds on NLSP abundances and lifetimes are much more stringent. In the case of dominant hadronic NLSP decays and lifetimes $\tau_{\mathrm{NLSP}} \gtrsim 10^{8} \mathrm{~s}$ one finds the upper bounds [51-53]

$$
\begin{array}{lll}
\Omega_{\mathrm{NLSP}} h^{2} \lesssim 1 \cdot 10^{-4} & \text { from } & { }^{2} \mathrm{H} \\
\Omega_{\mathrm{NLSP}} h^{2} \lesssim 3 \cdot 10^{-5} & \text { from } & { }^{3} \mathrm{He} .
\end{array}
$$

A detailed analysis for a general neutralino NLSP [12] has shown that, except for special points in parameter space, these constraints can only be satisfied for rather short NLSP 
lifetimes, $\tau_{\mathrm{NLSP}} \lesssim 10^{2}-10^{3} \mathrm{~s}$. For a gravitino mass of $100 \mathrm{GeV}$ this requires NLSP masses in excess of $2 \mathrm{TeV}$.

In our model the dominant NLSP decays are the 2-body decay into gravitino and photon, and the 3-body decay into gravitino and hadrons via a virtual $Z$-boson. The 3-body decay via an intermediate Higgs boson is suppressed by small Yukawa couplings, except possibly for a $\psi_{3 / 2} b \bar{b}$ final state (since $y_{b}$ is sizeable at large $\tan \beta$ ). In any case, $Z$-exchange dominates because there are many more final states available. For large $\tan \beta$ the corresponding decay widths are given by [12]

$$
\begin{aligned}
\Gamma\left(\chi_{1}^{0} \rightarrow \psi_{3 / 2} \gamma\right) & \simeq \frac{\epsilon_{h \gamma}^{2}}{48 \pi M_{\mathrm{P}}^{2}} \frac{\left(m_{\chi_{1}^{0}}\right)^{5}}{m_{3 / 2}^{2}}\left(1-\left(\frac{m_{\chi_{1}^{0}}}{m_{3 / 2}}\right)^{2}\right)^{3}\left(1+3\left(\frac{m_{\chi_{1}^{0}}}{m_{3 / 2}}\right)^{2}\right), \\
\Gamma\left(\chi_{1}^{0} \rightarrow \psi_{3 / 2} \text { had }\right) & \simeq \frac{r_{\text {had }, b \bar{b}}}{96(4 \pi)^{3} M_{\mathrm{P}}^{2}} \frac{\left(m_{\chi_{1}^{0}}\right)^{7}}{m_{3 / 2}^{2}} \frac{g_{2}^{2}}{M_{Z}^{2} c_{w}^{2}}\left(1-\frac{4}{3} s_{w}^{2}+\frac{8}{9} s_{w}^{4}\right) ;
\end{aligned}
$$

here $c_{w}=\cos \theta_{w}, s_{w}=\sin \theta_{w}, \theta_{w}$ is the weak mixing angle, $r_{\text {had }, b \bar{b}}=\operatorname{BR}(Z \rightarrow$ hadrons $) /$ $\operatorname{BR}(Z \rightarrow b \bar{b})$, and

$$
\epsilon_{h \gamma}=-\frac{s_{w} c_{w} M_{Z}\left(M_{2}-M_{1}\right)}{\sqrt{2} M_{1} M_{2}}
$$

is the higgsino-photino mixing angle. The ratio of branching fractions $r_{\text {had }, b \bar{b}}=4.6$ enters eq. (4.4) because we are using the decay width for $Z \rightarrow b \bar{b}$ in order to estimate the decay width into any hadronic final state.

In our model $\epsilon_{h \gamma}=-0.013$. Because of this small mixing the hadronic decays dominate. We find

$$
\Gamma^{-1}\left(\chi_{1}^{0} \rightarrow \psi_{3 / 2} \text { had }\right) \simeq 2 \cdot 10^{11} \mathrm{~s}, \quad \Gamma^{-1}\left(\chi_{1}^{0} \rightarrow \psi_{3 / 2} \gamma\right) \simeq 3 \cdot 10^{12} \mathrm{~s} .
$$

The $\chi_{1}^{0}$ relic abundance in our model is predicted to be rather small. This is because $\chi_{1}^{0}$ is nearly degenerate with $\chi_{1}^{ \pm}$in mass, so the $\chi_{1}^{0}$ can efficiently coannihilate with the chargino [49]. Using the micrOMEGAs code [54], we find for the spectrum of section 3.2 a neutralino relic abundance of

$$
\Omega_{\chi_{1}^{0}} h^{2}=3.2 \cdot 10^{-3}
$$

This is four orders of magnitude smaller than a typical bino relic density, but it still exceeds the BBN bounds (4.1), (4.2) by one to two orders of magnitude. This may be remedied in several ways, for instance by introducing small R-parity violating couplings [55] or by additional entropy production before nucleosynthesis [56]. Note also that, because of the small $\chi_{1}^{0}$ relic density of eq. (4.7), the lightest neutralino is not a possible dark matter candidate.

Our model predicts intriguing and distinctive signatures for the LHC experiments. The low energy particle spectrum contains only three fermions in addition to the Standard Model particles, two of them neutral and one charged, almost mass degenerate and close to the Higgs boson mass. The lightest neutralino will decay mostly outside the detector, even if R-parity is broken [57]. The heavier fermions decay into the next lighter one and a 
few hadrons via a virtual W-boson. The near mass degeneracy leads to rather long decay lengths of $c \tau_{\chi}=\mathcal{O}(1 \mu \mathrm{m})$. Energetic decaying higgsinos will lead to events with missing energy accompanied by low-momentum jets and leptons. These signatures, the production of charginos and neutralinos in cascades and Drell-Yan processes, and the predictions for rare processes will be discussed in a forthcoming paper.

\section{Conclusions}

We have studied the mediation of supersymmetry breaking in unified models with GUTsized extra dimensions. The MSSM soft terms receive contributions both from gravity mediation and from gauge mediation, since such models often contain suitable messenger fields with GUT-scale masses. These GUT-scale messengers tend to come in large numbers, and they do not have to form complete GUT multiplets. The presence of incomplete multiplets turns out to be crucial in achieving realistic electroweak symmetry breaking. Gauge mediation dominates, and as a consequence the superpartner spectrum is quite peculiar: The higgsinos can be as light as $100-200 \mathrm{GeV}$, because the $\mu$ term is induced by gravity mediation, while the remaining superpartners and the heavy Higgs bosons are much heavier. We have presented some sample spectra, and discussed the implications for naturalness and cosmology. As the lightest superparticle, the gravitino is a natural dark matter candidate. The relic abundance of the higgsino NLSP is substantially reduced by coannihilation, but there is still some tension remaining with the current bounds from primordial nucleosynthesis. A study of the resulting collider phenomenology is currently in preparation.

\section{Acknowledgments}

The authors thank L. Covi, J. Hasenkamp, S. Kraml, and R. Valandro for useful discussions.

Open Access. This article is distributed under the terms of the Creative Commons Attribution Noncommercial License which permits any noncommercial use, distribution, and reproduction in any medium, provided the original author(s) and source are credited.

\section{References}

[1] R. Blumenhagen, Gauge coupling unification in F-theory grand unified theories, Phys. Rev. Lett. 102 (2009) 071601 [arXiv:0812.0248] [SPIRES].

[2] J. Marsano, N. Saulina and S. Schäfer-Nameki, Compact F-theory GUTs with U(()1) $P Q$, JHEP 04 (2010) 095 [arXiv: 0912.0272] [SPIRES].

[3] A. Anandakrishnan and S. Raby, Gauge coupling unification in heterotic string models with gauge mediated SUSY breaking, Phys. Rev. D 83 (2011) 075008 [arXiv:1101.1976] [SPIRES].

[4] G.F. Giudice and A. Masiero, A natural solution to the mu problem in supergravity theories, Phys. Lett. B 206 (1988) 480 [SPIRES]. 
[5] CMS collaboration, V. Khachatryan et al., Search for supersymmetry in pp collisions at $7 \mathrm{TeV}$ in events with jets and missing transverse energy, Phys. Lett. B 698 (2011) 196 [arXiv:1101.1628] [SPIRES].

[6] CMS collaboration, S. Chatrchyan et al., Search for supersymmetry in pp collisions at $\sqrt{(s)}=7$ TeV in events with two photons and missing transverse energy, arXiv:1103.0953 [SPIRES].

[7] CMS collaboration, S. Chatrchyan et al., Search for neutral MSSM Higgs bosons decaying to tau pairs in pp collisions at $\sqrt{(s)}=7 \mathrm{TeV}$, arXiv:1104.1619 [SPIRES].

[8] ATLAS collaboration, G. Aad et al., Search for supersymmetry using final states with one lepton, jets and missing transverse momentum with the ATLAS detector in $\sqrt{(s)}=7$ TeV pp, Phys. Rev. Lett. 106 (2011) 131802 [arXiv:1102.2357] [SPIRES].

[9] ATLAS collaboration, J.B.G. da Costa et al., Search for squarks and gluinos using final states with jets and missing transverse momentum with the ATLAS detector in $\sqrt{(} s)=7$ TeV proton-proton collisions, Phys. Lett. B 701 (2011) 186 [arXiv:1102.5290] [SPIRES].

[10] ATLAS collaboration, G. Aad et al., Search for supersymmetry in pp collisions at $\sqrt{(s)}=7$ TeV in final states with missing transverse momentum and b-jets, arXiv: 1103.4344 [SPIRES].

[11] M. Bolz, W. Buchmüller and M. Plümacher, Baryon asymmetry and dark matter, Phys. Lett. B 443 (1998) 209 [hep-ph/9809381] [SPIRES].

[12] L. Covi, J. Hasenkamp, S. Pokorski and J. Roberts, Gravitino dark matter and general neutralino NLSP, JHEP 11 (2009) 003 [arXiv:0908.3399] [SPIRES].

[13] G.F. Giudice and R. Rattazzi, Theories with gauge-mediated supersymmetry breaking, Phys. Rept. 322 (1999) 419 [hep-ph/9801271] [SPIRES].

[14] Y. Kawamura, Gauge symmetry reduction from the extra space $S^{1} / Z_{2}$, Prog. Theor. Phys. 103 (2000) 613 [hep-ph/9902423] [SPIRES].

[15] Y. Kawamura, Split multiplets, coupling unification and extra dimension, Prog. Theor. Phys. 105 (2001) 691 [hep-ph/0012352] [SPIRES].

[16] L.J. Hall and Y. Nomura, Gauge unification in higher dimensions, Phys. Rev. D 64 (2001) 055003 [hep-ph/0103125] [SPIRES].

[17] A. Hebecker and J. March-Russell, A minimal $S^{1} /\left(Z_{2} \times Z_{2}^{\prime}\right)$ orbifold GUT, Nucl. Phys. B 613 (2001) 3 [hep-ph/0106166] [SPIRES].

[18] A. Hebecker and J. March-Russell, The structure of GUT breaking by orbifolding, Nucl. Phys. B 625 (2002) 128 [hep-ph/0107039] [SPIRES].

[19] T. Asaka, W. Buchmüller and L. Covi, Gauge unification in six dimensions, Phys. Lett. B 523 (2001) 199 [hep-ph/0108021] [SPIRES].

[20] T. Asaka, W. Buchmüller and L. Covi, Quarks and leptons between branes and bulk, Phys. Lett. B 563 (2003) 209 [hep-ph/0304142] [SPIRES].

[21] T. Kobayashi, S. Raby and R.-J. Zhang, Constructing 5d orbifold grand unified theories from heterotic strings, Phys. Lett. B 593 (2004) 262 [hep-ph/0403065] [SPIRES].

[22] T. Kobayashi, S. Raby and R.-J. Zhang, Searching for realistic $4 d$ string models with a Pati-Salam symmetry: Orbifold grand unified theories from heterotic string compactification on a $Z_{6}$ orbifold, Nucl. Phys. B 704 (2005) 3 [hep-ph/0409098] [SPIRES]. 
[23] S. Förste, H.P. Nilles, P.K.S. Vaudrevange and A. Wingerter, Heterotic brane world, Phys. Rev. D 70 (2004) 106008 [hep-th/0406208] [SPIRES].

[24] O. Lebedev et al., A mini-landscape of exact MSSM spectra in heterotic orbifolds, Phys. Lett. B 645 (2007) 88 [hep-th/0611095] [SPIRES].

[25] W. Buchmüller, K. Hamaguchi, O. Lebedev and M. Ratz, Supersymmetric standard model from the heterotic string, Phys. Rev. Lett. 96 (2006) 121602 [hep-ph/0511035] [SPIRES].

[26] W. Buchmüller, K. Hamaguchi, O. Lebedev and M. Ratz, Supersymmetric standard model from the heterotic string. II, Nucl. Phys. B 785 (2007) 149 [hep-th/0606187] [SPIRES].

[27] R. Donagi and M. Wijnholt, Model building with F-theory, arXiv:0802.2969 [SPIRES].

[28] R. Donagi and M. Wijnholt, Breaking GUT groups in F-theory, arXiv:0808.2223 [SPIRES].

[29] C. Beasley, J.J. Heckman and C. Vafa, GUTs and exceptional branes in F-theory - I, JHEP 01 (2009) 058 [arXiv: 0802.3391] [SPIRES].

[30] C. Beasley, J.J. Heckman and C. Vafa, GUTs and exceptional branes in F-theory - II: experimental predictions, JHEP 01 (2009) 059 [arXiv:0806.0102] [SPIRES].

[31] J. Marsano, N. Saulina and S. Schäfer-Nameki, Monodromies, Fluxes and Compact Three-Generation F-theory GUTs, JHEP 08 (2009) 046 [arXiv: 0906.4672] [SPIRES].

[32] J.L. Feng, C.G. Lester, Y. Nir and Y. Shadmi, The standard model and supersymmetric flavor puzzles at the Large Hadron Collider, Phys. Rev. D 77 (2008) 076002 [arXiv:0712.0674] [SPIRES].

[33] Y. Nomura, M. Papucci and D. Stolarski, Flavorful supersymmetry, Phys. Rev. D 77 (2008) 075006 [arXiv: 0712.2074] [SPIRES].

[34] Y. Nomura, M. Papucci and D. Stolarski, Flavorful supersymmetry from higher dimensions, JHEP 07 (2008) 055 [arXiv:0802.2582] [SPIRES].

[35] G. Hiller, Y. Hochberg and Y. Nir, Flavor changing processes in supersymmetric models with hybrid gauge- and gravity-mediation, JHEP 03 (2009) 115 [arXiv:0812.0511] [SPIRES].

[36] M. Ibe and R. Kitano, Sweet spot supersymmetry, JHEP 08 (2007) 016 [arXiv:0705.3686] [SPIRES].

[37] J. Marsano, N. Saulina and S. Schäfer-Nameki, Gauge mediation in F-theory GUT models, Phys. Rev. D 80 (2009) 046006 [arXiv:0808.1571] [SPIRES].

[38] E. Dudas, Y. Mambrini, S. Pokorski and A. Romagnoni, Moduli stabilization with Fayet-Iliopoulos uplift, JHEP 04 (2008) 015 [arXiv: 0711.4934] [SPIRES].

[39] E. Dudas, Y. Mambrini, S. Pokorski, A. Romagnoni and M. Trapletti, Gauge vs. gravity mediation in models with anomalous $\mathrm{U}(() 1)$ 's, JHEP 03 (2009) 011 [arXiv:0809.5064] [SPIRES].

[40] D.G. Cerdeno, Y. Mambrini and A. Romagnoni, Gravitino dark matter in hybrid gauge-gravity models, JHEP 11 (2009) 113 [arXiv:0907.4985] [SPIRES].

[41] A. De Simone, R. Franceschini, G.F. Giudice, D. Pappadopulo and R. Rattazzi, Lopsided gauge mediation, JHEP 05 (2011) 112 [arXiv:1103.6033] [SPIRES].

[42] B.C. Allanach, SOFTSUSY: a program for calculating supersymmetric spectra, Comput. Phys. Commun. 143 (2002) 305 [hep-ph/0104145] [SPIRES]. 
[43] E. Witten, Strong coupling expansion of Calabi-Yau compactification, Nucl. Phys. B 471 (1996) 135 [hep-th/9602070] [SPIRES].

[44] A. Hebecker and M. Trapletti, Gauge unification in highly anisotropic string compactifications, Nucl. Phys. B 713 (2005) 173 [hep-th/0411131] [SPIRES].

[45] W. Buchmüller, R. Catena and K. Schmidt-Hoberg, Small extra dimensions from the interplay of gauge and supersymmetry breaking, Nucl. Phys. B 804 (2008) 70 [arXiv: 0803.4501] [SPIRES].

[46] R. Barbieri and G.F. Giudice, Upper bounds on supersymmetric particle masses, Nucl. Phys. B 306 (1988) 63 [SPIRES].

[47] D. Horton and G.G. Ross, Naturalness and focus points with non-universal gaugino masses, Nucl. Phys. B 830 (2010) 221 [arXiv:0908.0857] [SPIRES].

[48] J.R. Ellis, G.B. Gelmini, J.L. Lopez, D.V. Nanopoulos and S. Sarkar, Astrophysical constraints on massive unstable neutral relic particles, Nucl. Phys. B 373 (1992) 399 [SPIRES].

[49] S. Mizuta and M. Yamaguchi, Coannihilation effects and relic abundance of Higgsino-dominant LSPs, Phys. Lett. B 298 (1993) 120 [hep-ph/9208251] [SPIRES].

[50] J. Edsjo and P. Gondolo, Neutralino relic density including coannihilations, Phys. Rev. D 56 (1997) 1879 [hep-ph/9704361] [SPIRES].

[51] M. Kawasaki, K. Kohri and T. Moroi, Hadronic decay of late-decaying particles and big-bang nucleosynthesis, Phys. Lett. B 625 (2005) 7 [astro-ph/0402490] [SPIRES].

[52] M. Kawasaki, K. Kohri and T. Moroi, Big-bang nucleosynthesis and hadronic decay of long-lived massive particles, Phys. Rev. D 71 (2005) 083502 [astro-ph/0408426] [SPIRES].

[53] K. Jedamzik, Big bang nucleosynthesis constraints on hadronically and electromagnetically decaying relic neutral particles, Phys. Rev. D 74 (2006) 103509 [hep-ph/0604251] [SPIRES].

[54] G. Bélanger, F. Boudjema, A. Pukhov and A. Semenov, MicrOMEGAs2.0: A program to calculate the relic density of dark matter in a generic model, Comput. Phys. Commun. 176 (2007) 367 [hep-ph/0607059] [SPIRES].

[55] W. Buchmüller, L. Covi, K. Hamaguchi, A. Ibarra and T. Yanagida, Gravitino dark matter in R-parity breaking vacua, JHEP 03 (2007) 037 [hep-ph/0702184] [SPIRES].

[56] J. Hasenkamp and J. Kersten, Leptogenesis, gravitino dark matter and entropy production, Phys. Rev. D 82 (2010) 115029 [arXiv:1008.1740] [SPIRES].

[57] S. Bobrovskyi, W. Buchmüller, J. Hajer and J. Schmidt, Broken R-parity in the sky and at the LHC, JHEP 10 (2010) 061 [arXiv: 1007.5007] [SPIRES]. 